\title{
. \\ Tirzepatide cardiovascular event risk assessment: a pre-specified meta-analysis
}

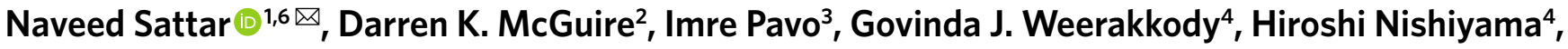 \\ Russell J. Wiese ${ }^{4}$ and Sophia Zoungas ${ }^{5,6} \times$
}

Tirzepatide is a novel, once weekly, dual GIP/GLP-1 receptor agonist and is under development for the treatment of type 2 diabetes (T2D) and obesity. Its association with cardiovascular outcomes requires evaluation. This pre-specified cardiovascular meta-analysis included all seven randomized controlled trials with a duration of at least 26 weeks from the tirzepatide T2D clinical development program, SURPASS. The pre-specified primary objective of this meta-analysis was the comparison of the time to first occurrence of confirmed four-component major adverse cardiovascular events (MACE-4; cardiovascular death, myocardial infarction, stroke and hospitalized unstable angina) between pooled tirzepatide groups and control groups. A stratified Cox proportional hazards model, with treatment as a fixed effect and trial-level cardiovascular risk as the stratification factor, was used for the estimation of hazard ratios (HRs) and confidence intervals (Cls) comparing tirzepatide to control. Data from 4,887 participants treated with tirzepatide and 2,328 control participants were analyzed. Overall, 142 participants, 109 from the trial with high cardiovascular risk and 33 from the six trials with lower cardiovascular risk, had at least one MACE-4 event. The HRs comparing tirzepatide versus controls were 0.80 (95\% Cl, 0.57-1.11) for $\mathrm{MACE}-4 ; 0.90$ (95\% Cl, 0.50-1.61) for cardiovascular death; and $0.80(95 \% \mathrm{Cl}, 0.51-1.25)$ for all-cause death. No evidence of effect modifications was observed for any subgroups, although the evidence was stronger for participants with high cardiovascular risk. Tirzepatide did not increase the risk of major cardiovascular events in participants with T2D versus controls.

$\mathrm{T}$ $2 \mathrm{D}$ is responsible for an average two-fold-higher risk for cardiovascular events, such as coronary heart disease, ischemic stroke, hospitalization for heart failure (HHF) and vascular death, independent of other established risk factors ${ }^{1,2}$.

Glucagon-like peptide-1 receptor agonists (GLP-1RAs) are now considered the first choice of injectable therapy for many people with T2D, with several members of the class having proven cardiovascular efficacy ${ }^{3}$. Building on that concept, the combined glucose-dependent insulinotropic polypeptide (GIP) and GLP1RAs have been proposed as a novel therapeutic option for $\mathrm{T}_{2} \mathrm{D}^{4}$. Tirzepatide is one such molecule that has shown marked glycemia and weight benefits in a series of trials ${ }^{5,6}$. For example, when compared to placebo, semaglutide $1 \mathrm{mg}$ per week, dulaglutide $1.5 \mathrm{mg}$ per week and insulin degludec or insulin glargine $100 \mathrm{U} \mathrm{ml}^{-1}$, tirzepatide was more effective in reducing glycated hemoglobin (HbA1c) and weight in people with T2D over a 26-52-week treatment period ${ }^{5,6}$. Tirzepatide might improve glycemic control beyond that of GLP-1RAs through direct and indirect actions on the pancreas and other tissues, including enhancing pancreatic $\beta$-cell insulin secretion, reducing glucose-adjusted glucagon secretion and improving insulin sensitivity beyond the levels usually explained by weight loss ${ }^{6-8}$. Additionally, tirzepatide's anorexigenic effect might exceed that of GLP-1RAs by integrating the activation signals of both GIP and GLP-1 receptor pathways in the brain $^{7,9}$. Among other beneficial effects, tirzepatide is also associated with improvements in lipoprotein profiles (more than GLP1RAs), blood pressure and several biomarkers of inflammation ${ }^{7,10}$. Notably, glycemic and weight effects appear to be maintained for at least 2 years while receiving tirzepatide, the longest observation period for this drug ${ }^{11}$.

Despite favorable effects of tirzepatide on a range of cardiovascular risk factors, to date its cardiovascular safety has been reported from results of only a single trial, SURPASS-4 (ref. ${ }^{11}$ ). This trial, which compared tirzepatide treatment to insulin glargine $100 \mathrm{U} \mathrm{ml}^{-1}$ treatment in people with T2D at increased cardiovascular risk, suggested no significant difference in the incidence of major cardiovascular events.

As required by the US Food and Drug Administration (FDA) and the European Medicines Agency (EMA) ${ }^{12-15}$, and to extend the safety evaluation in a broader population with T2D, the tirzepatide clinical development program for treatment of T2D (SURPASS) was also designed to evaluate the drug's cardiovascular safety in people with T2D at low, medium and high cardiovascular risk ${ }^{12}$. Therefore, a cardiovascular safety meta-analysis of data, from all phase 2 and phase 3 clinical trials with planned treatment duration of at least 26 weeks and at least one randomized comparator arm among adults with T2D, was conducted to assess the safety of tirzepatide with regard to major cardiovascular events relative to various randomized comparators.

Here we present the results of these pre-specified cardiovascular safety meta-analyses and selected post hoc exploratory analyses of interest, based on prospectively collected and centrally adjudicated MACE events.

\section{Results}

Data from one phase 2 trial, five international phase 3 trials and one regional phase 3 trial in Japan, each with at least one MACE- 4 event, were included in this meta-analysis (Supplementary Table 1).

'Institute of Cardiovascular and Medical Sciences, BHF Glasgow Cardiovascular Research Centre, University of Glasgow, Glasgow, UK. ${ }^{2}$ University of Texas Southwestern Medical Center and Parkland Health and Hospital System, Dallas, TX, USA. ${ }^{3}$ Eli Lilly Regional Operations GmbH, Vienna, Austria. ${ }^{4}$ Eli Lilly and Company, Indianapolis, IN, USA. ${ }^{5}$ School of Public Health and Preventative Medicine, Monash University, Melbourne, VC, Australia. ${ }^{6}$ These authors contributed equally: Naveed Sattar, Sophia Zoungas.凶e-mail: naveed.sattar@glasgow.ac.uk; sophia.zoungas@monash.edu 
Baseline demographics and clinical characteristics. A total of 7,215 randomized participants were included in the meta-analysis (pooled tirzepatide group treated with a mean assigned dose of $9.9 \mathrm{mg}$ per week of tirzepatide, $n=4,887(1 \mathrm{mg}, n=52 ; 5 \mathrm{mg}$, $n=1,608 ; 10 \mathrm{mg}, n=1,606 ; 15 \mathrm{mg}, n=1621)$ and pooled comparator group: $n=2,328$ (placebo, $n=286$; insulin degludec, $n=360$; insulin glargine, $n=1,000$, semaglutide $1 \mathrm{mg}, n=469$; dulaglutide $1.5 \mathrm{mg}$, $n=54$; dulaglutide $0.75 \mathrm{mg}, n=159$ ) (Table 1 ). The total study drug exposure was 4,404.3 patient-years in the pooled tirzepatide group and 2,470.7 patient-years in the pooled comparator group, wherein participants were exposed for a median duration of 44 weeks. The total duration of follow-up was 5,100.9 patient-years in the pooled tirzepatide group and 2,757.2 patient-years in the pooled comparator group, as the participants were followed-up for a median duration of 55.3 weeks. Premature trial discontinuations were reported in $321(6.7 \%)$ participants in the pooled tirzepatide group compared to $208(8.2 \%)$ participants in the pooled comparator group. Overall, the most frequently reported reasons for premature discontinuation from the study were similar across both pooled treatment groups and included withdrawal by participant (pooled tirzepatide group: $117(2.3 \%)$ participants as compared to the pooled comparator group: 70 (2.8\%) participants) and lost to follow-up (pooled tirzepatide group: $73(1.5 \%)$ participants as compared to the pooled comparator group: 47 (2.0\%) participants).

The baseline demographic characteristics were balanced across the pooled tirzepatide and pooled comparator group (Table 1). Overall, participants had a mean baseline HbAlc of $8.3 \%$, a mean diabetes duration of 9.3 years and a mean body mass index (BMI) of $32.8 \mathrm{~kg} \mathrm{~m}^{-2}$. The most frequently reported cardiovascular risk factors were hypertension and dyslipidemia $(4,627$ (73.9\%) participants had hypertension and 4,237 (67.7\%) participants had dyslipidemia) (Supplementary Table 2). In addition, 2,187 (34.9\%) participants had a history of cardiovascular disease. No clinically relevant differences were observed across the pooled treatment groups in the cardiovascular risk factors at baseline. At trial level, the major indices for high cardiovascular risk were markedly different. Notably, history of cardiovascular disease was present in $86.9 \%$ of participants from SURPASS-4, whereas the prevalence of such conditions in the other trials was $5.4-18.3 \%$ (Supplementary Table 3).

Cardiovascular safety meta-analysis. Results from time-to-event analysis of composite MACE-4 and individual components for the composite outcome included 142 participants with at least one MACE-4 (47 participants with cardiovascular deaths, 60 participants with myocardial infarction (MI) events, 30 participants with stroke events and 14 participants with hospitalized unstable angina (HUA)) and are presented in Fig. 1. Tirzepatide treatment was not associated with increased risk of the MACE- 4 outcome (HR of 0.80 (95\% CI, 0.57-1.11)) (Fig. 2 and Extended Data Fig. 1), cardiovascular death $(\mathrm{HR}=0.90(95 \% \mathrm{CI}, 0.50-1.61)), \mathrm{MI}(\mathrm{HR}=0.76(95 \%$ CI, 0.45-1.28)), stroke (HR $=0.81$ (95\% CI, 0.39-1.68)) and HUA $(\mathrm{HR}=0.46(95 \% \mathrm{CI}, 0.15-1.41))$. The rate of MACE-4 was markedly different among the individual trials in line with the differing cardiovascular risk profiles of trial participants. The highest rate was observed in SURPASS-4, 3.52 per 100 patient-years, with lower rates observed in the other trials, $0.26-1.13$ per 100 patient-years (Supplementary Table 4).

The results of the comparisons among the treatment groups for the MACE-3 outcomes demonstrated an HR of 0.83 (95\% CI, $0.58-1.18$ ) for MACE-3; an HR of 0.78 (95\% CI, 0.56-1.08) for composite outcome of MACE-3 or HHF; and an HR of 0.67 (95\% CI, 0.26-1.70) for HHF. All-cause death had an HR of 0.80 (95\% CI, $0.51-1.25$ ) (Fig. 1).

Time to first occurrence of confirmed MACE- 4 for trials including tirzepatide versus insulin glargine, insulin degludec and placebo combined demonstrated an HR of 0.73 (95\% CI, 0.51-1.05)
$(P=0.089)$ (Fig. 3a and Extended Data Fig. 1). Time to first occurrence of confirmed MACE-4 for SURPASS-4 only (tirzepatide versus insulin glargine in a high-cardiovascular-risk population) demonstrated an HR of 0.74 (95\% CI, 0.51-1.08) $(P=0.123)$ (Extended Data Fig. 1).

In the trials included in this meta-analysis, 85 participants underwent at least one confirmed coronary revascularization procedure during the study: 60 participants (ten surgical and 51 percutaneous) were classified as undergoing an urgent procedure, and 30 participants (ten surgical and 21 percutaneous) were classified as undergoing a non-urgent procedure. Additionally, 182 participants suffered a first occurrence of MACE-6 - the composite of MACE-4 plus HHF or coronary revascularizations. Post hoc analyses of the comparisons among the treatment groups for coronary revascularizations demonstrated an HR of 0.76 (95\% CI, 0.49-1.17), including 0.67 (95\% CI, 0.40-1.13) for urgent revascularizations and 0.77 (95\% CI, 0.37-1.61) for non-urgent revascularizations. MACE-6 had an HR of 0.79 (95\% CI, 0.58-1.06) (Fig. 4).

Subgroup analyses for the primary outcome of MACE- 4 by sex, age, baseline $\mathrm{HbA} 1 \mathrm{c}$, race, US or non-US clinical sites and baseline sodium glucose co-transporter 2 inhibitor (SGLT-2i) use demonstrated no significant effect modification (all $P_{\text {interaction }}>0.1$ ) (Fig. 5).

Before the completion of SURPASS-4 and SURPASS J-mono, an interim analysis was performed for regulatory purposes. This analysis included 116 participants who had experienced at least one component of the MACE- 4 composite endpoint. Once weekly tirzepatide and treatment exposure of up to 104 weeks (median follow-up of 55 weeks) resulted in an HR of 0.81 (97.85\% CI, $0.52-1.26$ ) for the pooled tirzepatide group compared to the pooled comparator group from 116 composite MACE- 4 endpoints (pooled tirzepatide: 60 (1.37 per 100 person-years) and pooled comparator: 56 (1.60 per 100 person-years)) and, therefore, met the criteria that treatment with tirzepatide was not associated with excess cardiovascular risk and initiated early close-out of SURPASS- 4 .

\section{Discussion}

This prospectively planned, pooled individual participant data, cardiovascular safety meta-analysis was conducted for the tirzepatide T2D clinical development program, SURPASS. The overall cardiovascular findings indicate that tirzepatide treatment given for a median duration of just over 1 year, at a mean randomization dose of $9.9 \mathrm{mg}$ per week to a population with just over one-third having established cardiovascular disease, is not associated with increased cardiovascular risk when compared to placebo or comparators not known to be cardioprotective, with point estimates of $\mathrm{HR}<1.0$ and upper confidence limits of $95 \% \mathrm{CI}<1.8$ for all MACE components. Furthermore, the incidence of MACE- 4 with tirzepatide starts to diverge from the control groups after approximately 1 year. A similar pattern was observed in most previous GLP-1RA cardiovascular outcomes trials (CVOTs) ${ }^{16-18}$.

The population in the SURPASS clinical development program included a wide spectrum of people on the continuum of T2Dfrom participants treated with diet and exercise alone with a mean duration of diabetes of 4.7 years (SURPASS-1) to participants for whom basal insulin treatment was insufficient with a mean duration of diabetes of 13.3 years (SURPASS-5). Similarly, participants' cardiovascular risk varied among trials. The SURPASS-4 trial enrolled participants at especially high cardiovascular risk, with enrichment criteria including previous cardiovascular event, established atherosclerotic cardiovascular disease and/or chronic kidney disease or heart failure. Consequently, as anticipated in the planning of the cardiovascular safety meta-analysis, this trial had the highest MACE incidence and contributed considerably to events analyzed in the present study. On the other hand, as similar changes in cardiovascular surrogate markers (HbA1c, weight, blood pressure and lipoproteins) by tirzepatide were observed in studies with 
Table 1 | Baseline demographics and clinical characteristics

\begin{tabular}{|c|c|c|c|}
\hline Parameter & All tirzepatide $N=4,887$ & All comparator $N=2,328$ & Total $N=7,215$ \\
\hline Age, years & $58.7(9.9)$ & $59.0(10.0)$ & $58.8(9.9)$ \\
\hline \multicolumn{4}{|l|}{ Age category, $n(\%)$} \\
\hline$\geq 65$ years & $1,466(31.5)$ & $832(32.3)$ & $2,298(31.9)$ \\
\hline Sex, female, $n(\%)$ & $2,163(43.8)$ & $962(42.6)$ & $3,125(43.3)$ \\
\hline \multicolumn{4}{|l|}{ Ethnicity, $n(\%)$} \\
\hline Not Hispanic or Latino & $2,212(45.8)$ & $1,091(45.6)$ & $3,303(45.8)$ \\
\hline Not reported & $636(11.9)$ & $217(11.7)$ & $853(11.8)$ \\
\hline \multicolumn{4}{|l|}{ Race, $n(\%)$} \\
\hline American Indian or Alaska Native & $356(7.4)$ & $164(6.7)$ & $520(7.2)$ \\
\hline Asian & $792(15.1)$ & $273(14.1)$ & $1,065(14.8)$ \\
\hline White & $3,507(72.6)$ & $1,783(75.1)$ & $5,290(73.4)$ \\
\hline \multicolumn{4}{|l|}{ Country, n (\%) } \\
\hline United States & $1,084(21.7)$ & $503(22.9)$ & $1,587(22.0)$ \\
\hline Outside United States & $3,803(78.3)$ & $1,825(77.1)$ & $5,628(78.0)$ \\
\hline Weight, kg & $91.12(20.32)$ & $90.59(20.36)$ & $90.98(20.37)$ \\
\hline $\mathrm{BMl}, \mathrm{kg} \mathrm{m}^{-2}$ & $32.849(6.263)$ & $32.673(6.259)$ & $32.791(6.262)$ \\
\hline Duration of diabetes, years & $9.23(6.81)$ & $9.22(6.78)$ & $9.26(6.83)$ \\
\hline $\mathrm{HbA1c}, \%$ & $8.30(0.94)$ & $8.27(0.91)$ & $8.29(0.93)$ \\
\hline \multicolumn{4}{|l|}{ HbA1c category, $n(\%)$} \\
\hline Triglycerides, $\mathrm{mg} \mathrm{dl}^{-1}$ & $185.67(131.72)$ & $181.61(134.30)$ & $184.53(133.16)$ \\
\hline Total cholesterol, mg dl-1 & $173.18(41.76)$ & $173.90(42.02)$ & $173.40(41.88)$ \\
\hline Smoking history, yes, $n$ (\%) & $1,453(30.7)$ & 719 (28.9) & $2,172(30.1)$ \\
\hline Current smoking status, yes, $n(\%)$ & $822(16.6)$ & $416(18.5)$ & $1238(17.2)$ \\
\hline UACR, $\mathrm{mg} \mathrm{g}^{-1}$, median & 11.0 & 12.0 & 12.0 \\
\hline \multicolumn{4}{|l|}{ UACR category, $n(\%)$} \\
\hline Microalbuminuria & $1,156(24.2)$ & $560(23.4)$ & $1,716(24.0)$ \\
\hline Macroalbuminuria & $258(5.6)$ & $123(4.6)$ & $381(5.3)$ \\
\hline $\mathrm{eGFR}, \mathrm{ml} / \mathrm{min} / 1.73 \mathrm{~m}^{2}$ & $89.02(18.88)$ & $88.99(18.60)$ & $89.04(18.76)$ \\
\hline \multicolumn{4}{|l|}{ eGFR category, $n$ (\%) } \\
\hline$<60 \mathrm{ml} / \mathrm{min} / 1.73 \mathrm{~m}^{2}$ & $363(8.4)$ & $233(8.2)$ & $596(8.3)$ \\
\hline$\geq 60 \mathrm{ml} / \mathrm{min} / 1.73 \mathrm{~m}^{2}$ & $4,523(91.6)$ & $2,094(91.8)$ & $6,617(91.7)$ \\
\hline
\end{tabular}

Data are presented as mean (s.d.) unless otherwise indicated (mITT population). Percentage is based on the number of participants with non-missing measurement at baseline. Data are strata size adjusted estimate. Strata are defined as trial-level cardiovascular risk (SURPASS-4 forms one stratum, and all other trials form one stratum). eGFR, estimated glomerular filtration rate; HDL, high-density lipoprotein; LDL, low-density lipoprotein; $N$, number of participants in the population; n, number of participants in the specified category; UACR, urine albumin to creatinine ratio.

high or moderate cardiovascular risk, it was assumed that the relative treatment effect would not be different regardless of the participants' baseline cardiovascular risk level ${ }^{6,11,19}$. Moreover, baseline cardiovascular risk did not modify the estimate of treatment effects of GLP-1RAs on MACE ${ }^{20}$.
The comparators across the trials' program were diverse. Some of the comparators, such as GLP-1RAs dulaglutide and semaglutide, have proven cardiovascular benefits as reflected in their product-labeled indications and endorsement across professional society guidelines and recommendations ${ }^{16,17,21}$, which would attenu- 


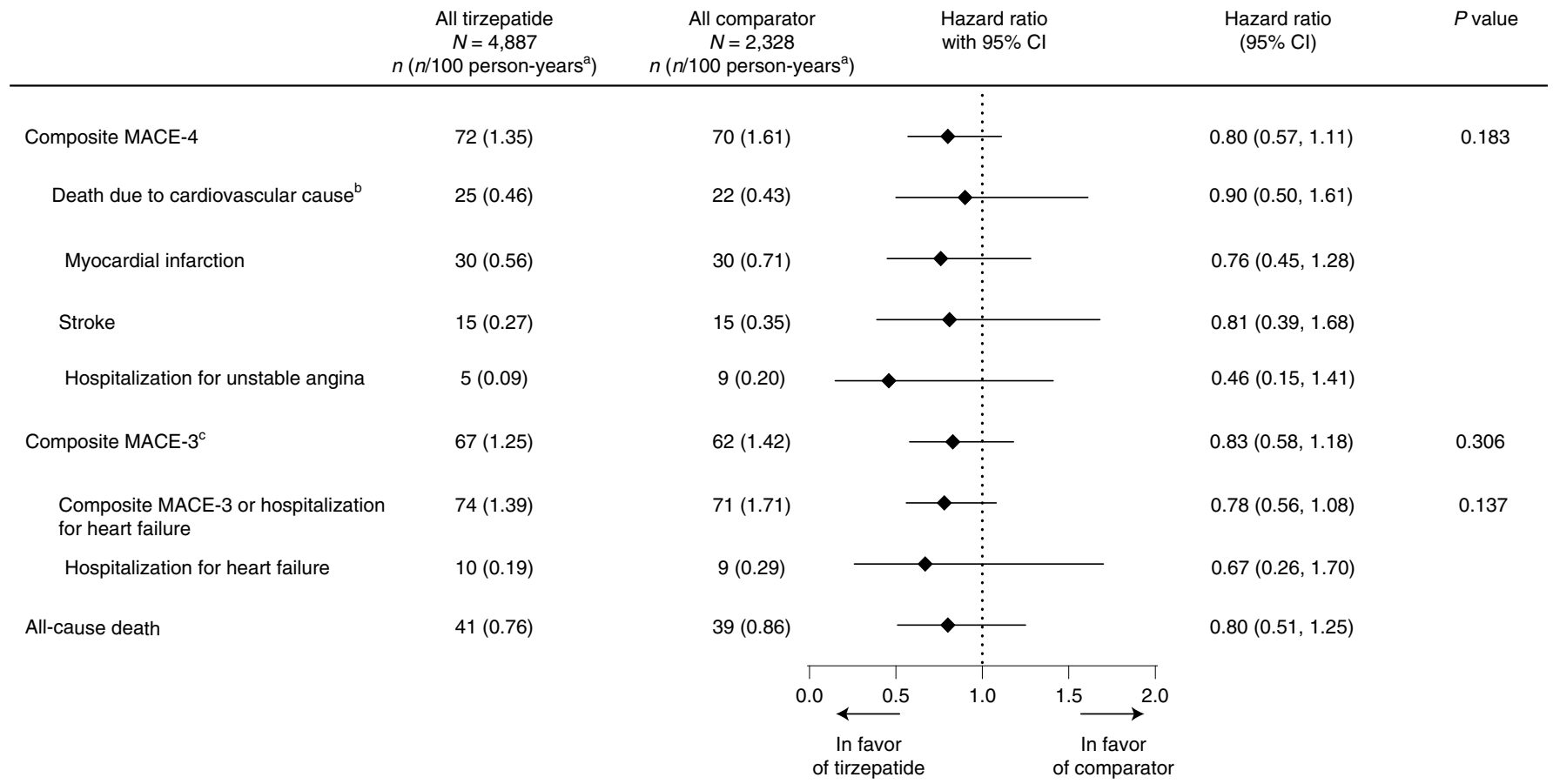

Fig. 1 | Primary and secondary cardiovascular outcomes confirmed by central-blinded adjudication. Data are point estimates of HR (illustrated by the

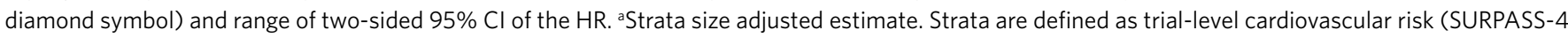
forms one stratum, and all other trials form one stratum) b ${ }^{\text {Death }}$ due to cardiovascular cause includes adjudication-confirmed death due to cardiovascular or undetermined cause. 'MACE-3 includes death due to cardiovascular or undetermined cause, MI and stroke. Note: $P$ values were based on the Wald chi-square test. $n$, number of participants in the specified category.

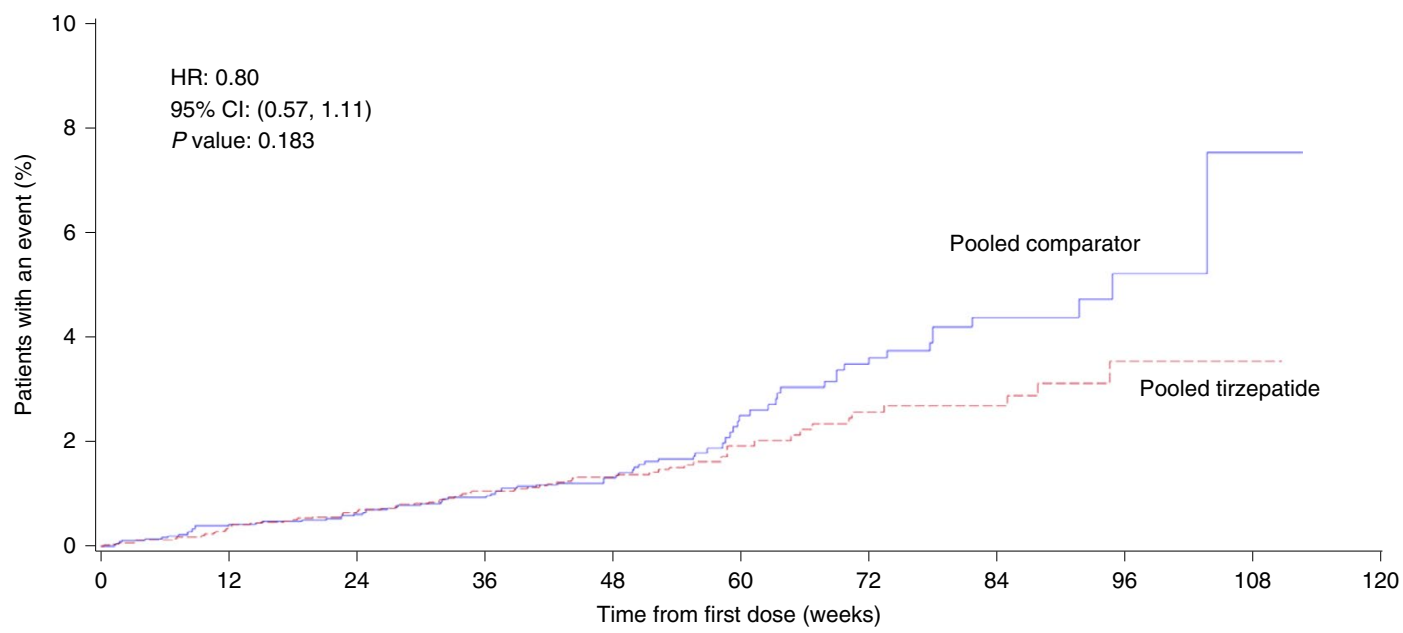

Planned follow-up period GPGB
SURPASS-1, -2 and -5
SURPASS-3 and J-mono (30 weeks) SURPASS- $1,-2$ and -5
SURPASS-3 and J-mono
SURPASS-4

(56-108 weeks)

\begin{tabular}{|c|c|c|c|c|c|c|c|c|c|}
\hline $15: 4813$ & 28:4726 & 43:4477 & 53:2477 & $62: 960$ & 68:832 & 69:515 & 72:188 & $72: 19$ & 72:0 \\
\hline 13:2292 & $19: 2250$ & 28:2118 & $36: 1438$ & $52: 914$ & $62: 794$ & $67: 496$ & $69: 172$ & $70: 14$ & \\
\hline
\end{tabular}

Fig. 2 | Adjusted Kaplan-Meier plot of pooled tirzepatide versus pooled comparator effect on time to first occurrence of adjudication-confirmed MACE-4 (primary outcome). Gray bars represent the planned follow-up period for trials GPGB (30 weeks); SURPASS-1, SURPASS-2 and SURPASS-5 (44 weeks); SURPASS-3 and SURPASS J-mono (56 weeks); and SURPASS-4 (56-108 weeks). Note: P values were based on the Wald chi-square test.

ate the comparative estimate of cardiovascular effect of tirzepatide. It was assumed, however, that any such effect would be negligible due to the short treatment duration of pooled trials and apparent lag time required to observe MACE benefits with dulaglutide or semaglutide (as reported by the placebo-controlled trials ${ }^{16,22}$ ). Placebo and the basal insulins included in the trials are considered to be neutral 


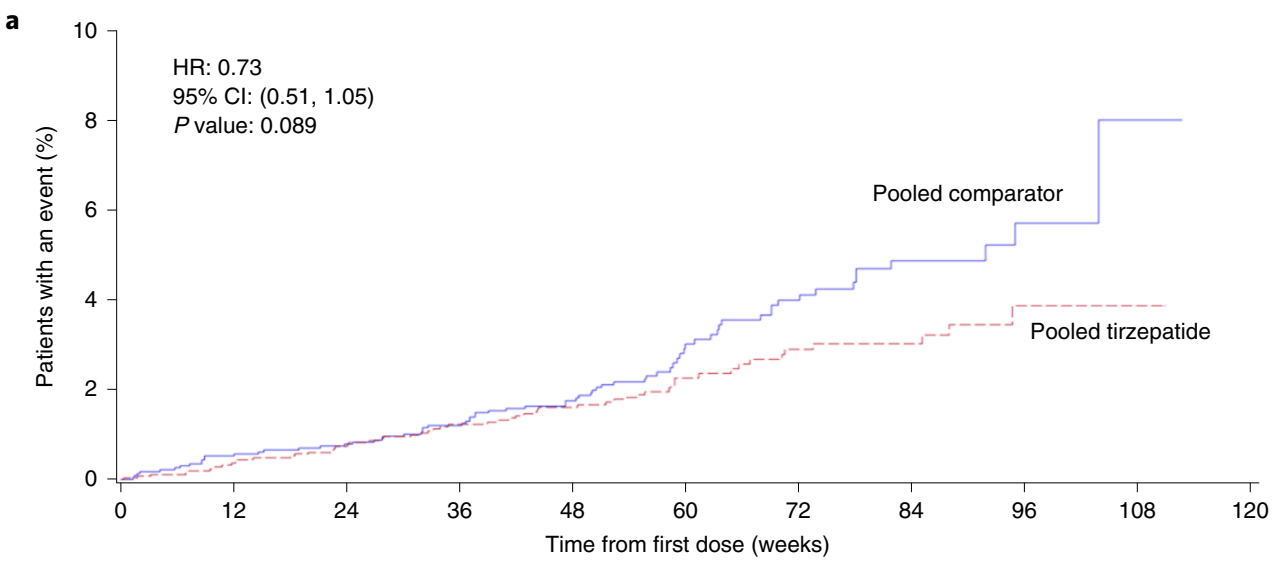

Planned follow-up period

GPGB (30 weeks)

SURPASS-1 and -5 (44 weeks)

$\begin{array}{ll}\text { SURPASS- } 3 & \text { (56 weeks) }\end{array}$

SURPASS-4

(56 weeks)

Cumulative number of events: number of patients at risk

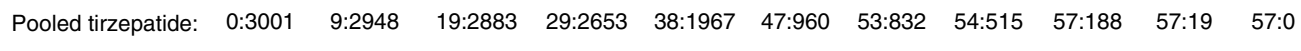

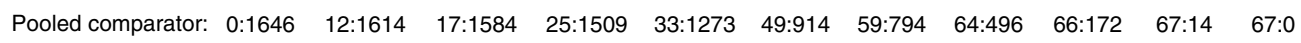

b

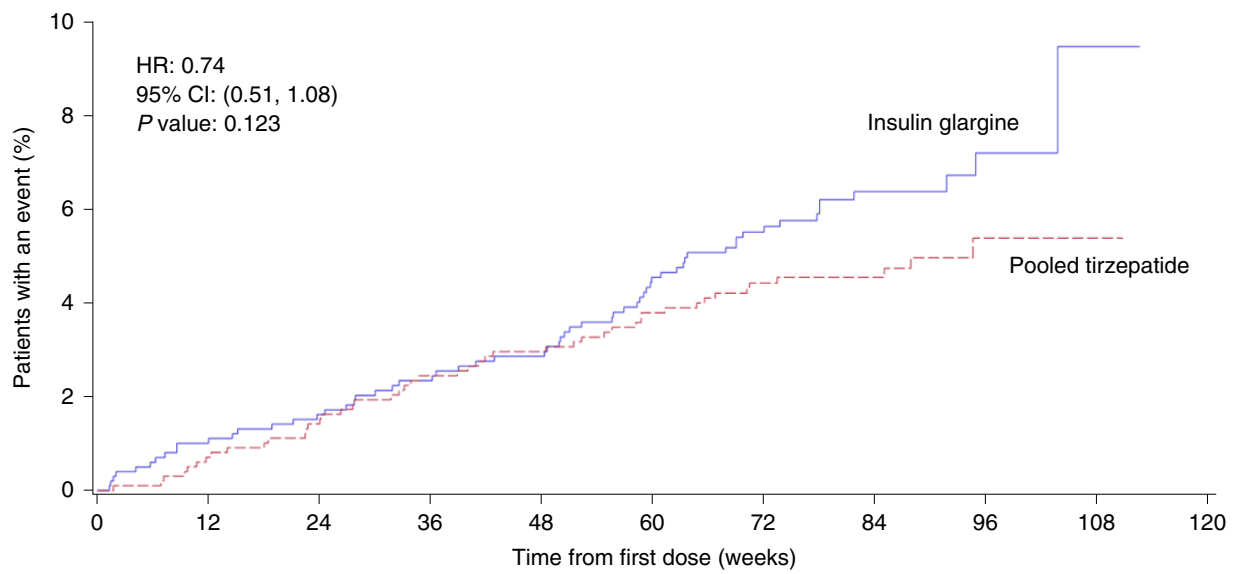

Planned follow-up period SURPASS-4

(56-108 weeks)

Cumulative number of events: number of patients at risk

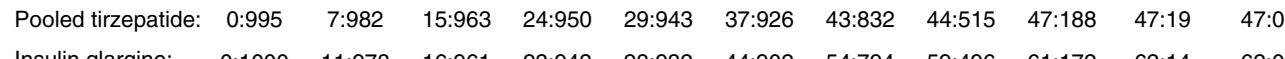

Fig. 3 | Time to first occurrence of adjudication-confirmed MACE-4 by trial groupings. Adjusted Kaplan-Meier estimates. a, Time to first occurrence of composite MACE-4, pooled tirzepatide versus insulin glargine, insulin degludec and placebo combined and mITT population. Gray bars represent the planned follow-up period for trials GPGB (30 weeks); SURPASS-1 and SURPASS-5 (44 weeks); and SURPASS-3 (56 weeks). b, Time to first occurrence of adjudicated-confirmed MACE-4, pooled tirzepatide versus insulin glargine, SURPASS-4 only and mITT population. Gray bar represents the planned follow-up period for SURPASS-4 (56-108 weeks). Note: $P$ values were based on the Wald chi-square test.

with regard to cardiovascular risk $^{18,21}$, with pre-specified analyses of this subset of comparators likely to more accurately reflect the cardiovascular safety of tirzepatide. Both subset analyses, including an analysis of the SURPASS-4 trial only and an analysis of all insulin and placebo comparator trials, were generally consistent with the MACE-4 result of the entire pooled population. Collectively, these data meet the regulatory cardiovascular safety criterion for consideration of a novel anti-hyperglycemic medication for initial approval, statistically excluding the upper confidence limit of the $95 \%$ CI of 1.8. Furthermore, some of these findings provide optimism for the potential positive cardiovascular effects of tirzepatide (aiming for a maximum dose of $15 \mathrm{mg}$ per day) being tested in the ongoing outcome trial SURPASS-CVOT (NCT04255433) ${ }^{23}$.

All individual trials had a balanced distribution of cardiovascular risk factors between pooled tirzepatide and control groups. However, this was not the case when participants were pooled from all trials. In SURPASS-4 (that is, participants with high cardiovascular risk), there was a higher ratio of participants in the control arm who had cardiovascular risk factors due to the 1:1 randomization scheme between the tirzepatide and insulin glargine groups. Therefore, these meta-analyses were based on pre-specified estimates adjusted for different randomization ratios between pooled tirzepatide versus pooled control in 


\begin{tabular}{cccc} 
All tirzepatide & All comparator & Hazard ratio & Hazard ratio \\
$N=4,887$ & $N=2,328$ & with $95 \% \mathrm{Cl}$ & $(95 \% \mathrm{Cl})$ \\
$n\left(n / 100\right.$ person-years $\left.^{\mathrm{a}}\right)$ & $n\left(n / 100\right.$ person-years $\left.^{\mathrm{a}}\right)$ & & \\
\hline
\end{tabular}

$\begin{array}{lll}\text { Coronary revascularization } & 42(0.78) & 43(1.03) \\ \text { Urgent coronary revascularization } & 28(0.52) & 32(0.79) \\ \text { Urgent percutaneous coronary intervention } & 22(0.41) & 29(0.73) \\ \text { Non-urgent coronary revascularization } & 15(0.28) & 15(0.35) \\ \text { Non-urgent percutaneous coronary intervention } & 12(0.22) & 9(0.20) \\ \text { MACE-6 } & & 89(2.14)\end{array}$

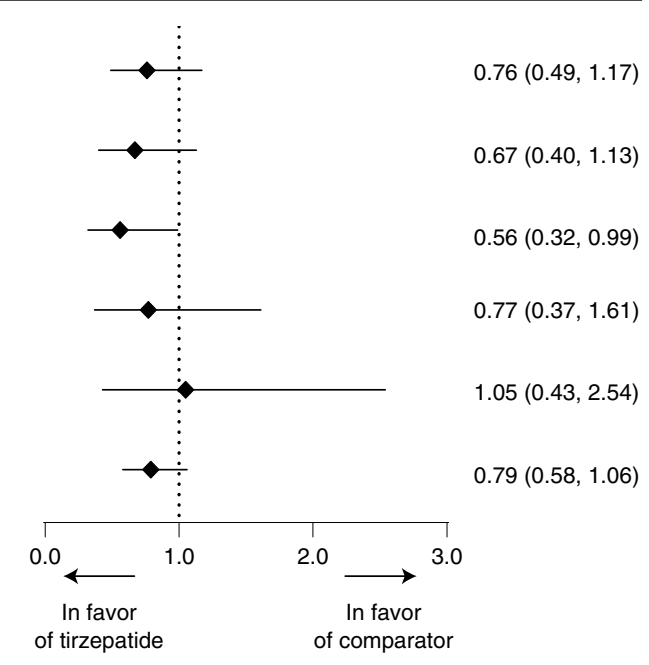

Fig. 4 | Other adjudication-confirmed cardiovascular outcomes. Data are point estimates of HR (illustrated by the diamond symbol) and range of two-sided $95 \% \mathrm{Cl}$ of the HR. aStrata size adjusted estimate. Strata are defined as trial-level cardiovascular risk (SURPASS-4 forms one stratum, and all other trials form one stratum). ${ }^{b}$ MACE- 6 includes MACE- 3 and all other adjudication-confirmed cardiovascular outcomes: HUA, HHF and coronary revascularization. $n$, number of participants in the specified category.

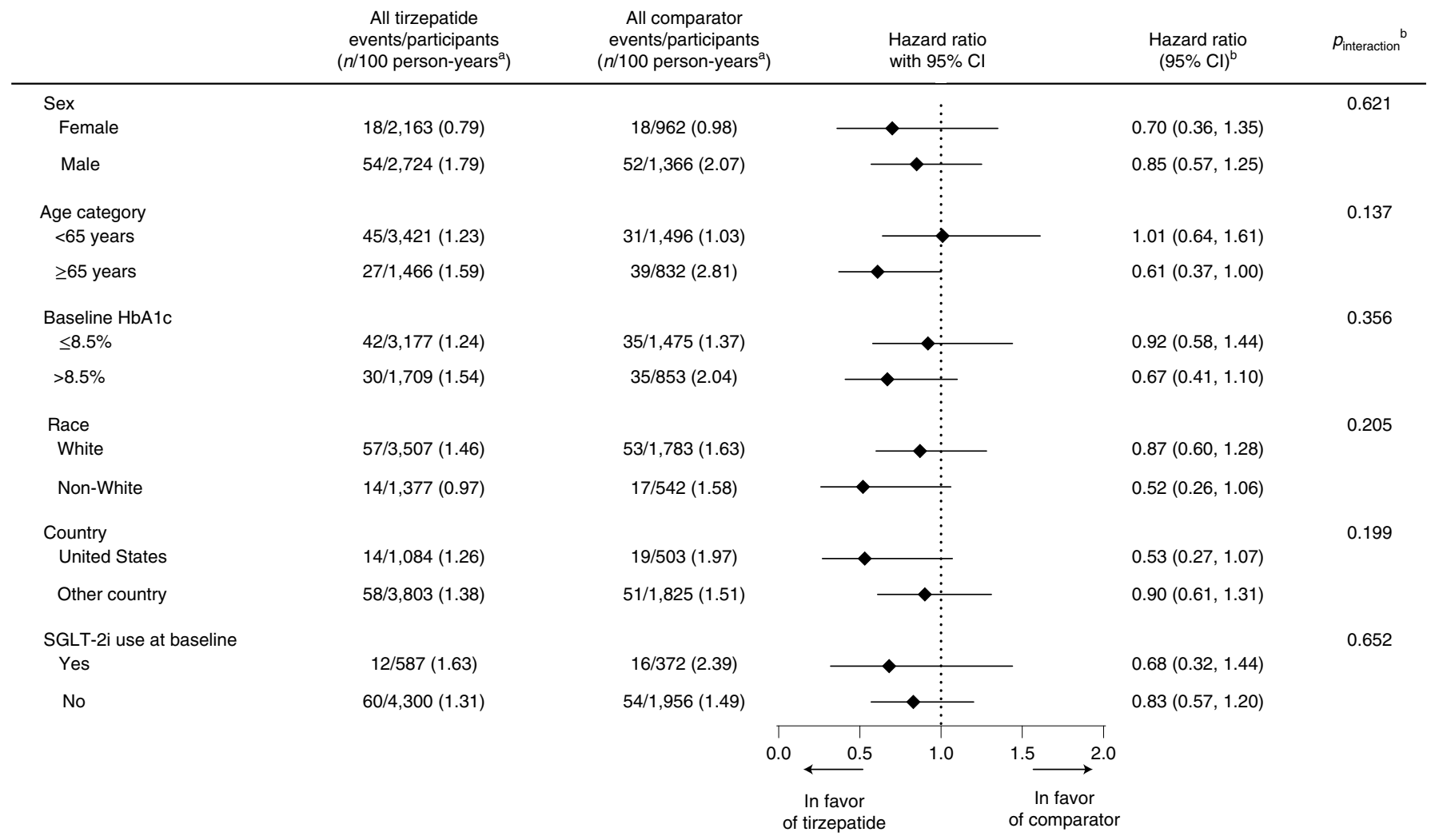

Fig. 5 | Subgroup analyses of the effects of tirzepatide on the adjudication-confirmed composite MACE-4. Data are point estimates of HR (illustrated by the diamond symbol) and range of two-sided $95 \% \mathrm{Cl}$ of the HR. ${ }^{\text {SStrata }}$ size adjusted estimate. Strata are defined as trial-level cardiovascular risk (SURPASS-4 forms one stratum, and all other trials form one stratum). ${ }^{b}$ Derived from a Cox proportional hazards model with treatment (pooled tirzepatide versus pooled comparator), the subgroup variable and the treatment-by-subgroup interaction term as fixed effects, stratified by study-level cardiovascular risk (SURPASS-4 forms one stratum, and all other trials form one stratum) for subgroups other than stratum. Pre-specified subgroup analyses were by sex, age and baseline $\mathrm{HbA1c}$; the rest of the analyses were post hoc. $P$ value is from the Wald chi-square test. $N$, number of participants in the subgroup in population; $n$, number of participants in the specified category. 
different trial-level cardiovascular risk strata. Without adjustment, the control group would be enriched with high-cardiovascular-risk participants, subject to more MACE, and would thus bias the results toward a lower HR in favor of tirzepatide.

In addition to the pre-specified cardiovascular analyses, post hoc analysis of the incidence of adjudication-confirmed coronary revascularizations was also assessed. The HRs overall, as well as in the sub-analysis stratified by urgent and non-urgent coronary revascularizations, were generally consistent with the primary and secondary cardiovascular safety findings. Moreover, the findings of the post hoc MACE- 6 analysis, including first occurrence of all adjudication-confirmed cardiac events that yielded a larger number of events for analysis compared to MACE- 4 or MACE-3, were broadly in line with the main cardiovascular findings.

The proportion of participants who were missing MACE assessments at the end of each trial was similar to contemporary glycemic trials and slightly higher than single CVOTs with MACE as the primary endpoints $\left(3-4 \%\right.$ at the maximum $\left.{ }^{16,22,24,25}\right)$. The missing assessments due to adverse events were balanced among pooled treatment groups; therefore, missing MACE assessments were considered missing at random, and the potential biases introduced due to missing data for the cardiovascular endpoint were likely minimal.

The present cardiovascular meta-analyses include a broad clinical population at different stages of their T2D course. The inclusion of a large number of participants with a history of multiple cardiovascular risk factors, prior events and prevalent cardiovascular disease, and participants with a trial treatment duration of up to 24 months, supported the assessment of cardiovascular safety ${ }^{26-35}$. Relatively few MACE were reported in participants with lower cardiovascular risk up to 56 weeks of follow-up despite high total investigational product exposures. Thus, cardiovascular safety assessment of tirzepatide was less robust in this subgroup.

Similarly to other clinical development programs of anti-hyperglycemic therapies for T2D, a limitation of these cardiovascular meta-analyses was the inclusion of tirzepatide clinical trials that excluded participants with recent unstable cardiovascular disease (for example, New York Heart Association class IV heart failure and recent cardiovascular events).

In conclusion, treatment with once weekly tirzepatide at the doses of $5 \mathrm{mg}, 10 \mathrm{mg}$ and $15 \mathrm{mg}$, with controlled treatment exposure of up to 104 weeks, was not associated with increased risk for cardiovascular events in people with T2D across a spectrum of T2D duration and cardiovascular risk levels.

\section{Online content}

Any methods, additional references, Nature Research reporting summaries, source data, extended data, supplementary information, acknowledgements, peer review information; details of author contributions and competing interests; and statements of data and code availability are available at https://doi.org/10.1038/ s41591-022-01707-4.

Received: 15 November 2021; Accepted: 19 January 2022; Published online: 24 February 2022

\section{References}

1. Benjamin, E. J. et al. Heart disease and stroke statistics-2018 update: a report from the American Heart Association. Circulation 137, e67-e492 (2018)

2. Rawshani, A. et al. Risk factors, mortality, and cardiovascular outcomes in patients with type 2 diabetes. N. Engl. J. Med. 379, 633-644 (2018).

3. Kristensen, S. L. et al. Cardiovascular, mortality, and kidney outcomes with GLP-1 receptor agonists in patients with type 2 diabetes: a systematic review and meta-analysis of cardiovascular outcome trials. Lancet Diabetes Endocrinol. 7, 776-785 (2019).

4. Holst, J. J. \& Rosenkilde, M. M. GIP as a therapeutic target in diabetes and obesity: insight from incretin co-agonists. J. Clin. Endocrinol. Metab. 105, e2710-e2716 (2020)
5. Rosenstock, J. et al. Efficacy and safety of a novel dual GIP and GLP-1 receptor agonist tirzepatide in patients with type 2 diabetes (SURPASS-1): a double-blind, randomised, phase 3 trial. Lancet 398, 143-155 (2021).

6. Frias, J. P. et al. Tirzepatide versus semaglutide once weekly in patients with type 2 diabetes. N. Engl. J. Med. 385, 503-515 (2021).

7. Frias, J. P. et al. Efficacy and safety of LY3298176, a novel dual GIP and GLP-1 receptor agonist, in patients with type 2 diabetes: a randomised, placebo-controlled and active comparator-controlled phase 2 trial. Lancet 392, 2180-2193 (2018).

8. Thomas, M. K. et al. Dual GIP and GLP-1 receptor agonist tirzepatide improves beta-cell function and insulin sensitivity in type 2 diabetes. J. Clin. Endocrinol. Metab. 106, 388-396 (2021).

9. Coskun, T. et al. LY3298176, a novel dual GIP and GLP-1 receptor agonist for the treatment of type 2 diabetes mellitus: from discovery to clinical proof of concept. Mol. Metab. 18, 3-14 (2018).

10. Wilson, J. M. et al. The dual glucose-dependent insulinotropic peptide and glucagon-like peptide-1 receptor agonist, tirzepatide, improves lipoprotein biomarkers associated with insulin resistance and cardiovascular risk in patients with type 2 diabetes. Diabetes Obes. Metab. 22, 2451-2459 (2020).

11. Del Prato, S. et al. Tirzepatide versus insulin glargine in type 2 diabetes and increased cardiovascular risk (SURPASS-4): a randomised, open-label, parallel-group, multicentre, phase 3 trial. Lancet 398, 1811-1824 (2021).

12. US Food anrd Dug Administration. Guidance for industry. Diabetes mellitus-evaluating cardiovascular risk in new antidiabetic therapies to treat type 2 diabetes. https://www.fda.gov/media/71297/download (2008).

13. European Medicines Agency, Committee for Medicinal Products for Human Use (CHMP). Guideline on clinical investigation of medicinal products in the treatment of diabetes mellitus. https://www.ema.europa.eu/en/documents/ scientific-guideline/draft-guideline-clinical-investigation-medicinal-productstreatment-prevention-diabetes-mellitus_en.pdf (2010).

14. US Food and Drug Administration. Guidance for Industry. Type 2 diabetes mellitus: evaluating the safety of new drugs for improving glycemic control. https://www.fda.gov/media/135936/download (2020).

15. Gore, M. O. \& McGuire, D. K. Cardiovascular disease and type 2 diabetes mellitus: regulating glucose and regulating drugs. Curr. Cardiol. Rep. 11, 258-263 (2009)

16. Gerstein, H. C. et al. Dulaglutide and cardiovascular outcomes in type 2 diabetes (REWIND): a double-blind, randomised placebo-controlled trial. Lancet 394, 121-130 (2019).

17. Husain, M. et al. Oral semaglutide and cardiovascular outcomes in patients with type 2 diabetes. N. Engl. J. Med. 381, 841-851 (2019).

18. Marso, S. P. et al. Efficacy and safety of degludec versus glargine in type 2 diabetes. N. Engl. J. Med. 377, 723-732 (2017).

19. Ludvik, B. et al. Once-weekly tirzepatide versus once-daily insulin degludec as add-on to metformin with or without SGLT2 inhibitors in patients with type 2 diabetes (SURPASS-3): a randomised, open-label, parallel-group, phase 3 trial. Lancet 398, 583-598 (2021).

20. Sattar, N. et al. Cardiovascular, mortality, and kidney outcomes with GLP-1 receptor agonists in patients with type 2 diabetes: a systematic review and meta-analysis of randomised trials. Lancet Diabetes Endocrinol. 9, 653-662 (2021).

21. ORIGIN Trial Investigatorset al. Basal insulin and cardiovascular and other outcomes in dysglycemia. N. Engl. J. Med. 367, 319-328 (2012).

22. Marso, S. P. et al. Semaglutide and cardiovascular outcomes in patients with type 2 diabetes. N. Engl. J. Med. 375, 1834-1844 (2016).

23. A Study of Tirzepatide (LY3298176) Compared With Dulaglutide on Major Cardiovascular Events in Participants With Type 2 Diabetes (SURPASS-CVOT). https://clinicaltrials.gov/ct2/show/NCT04255433

24. Zinman, B. et al. Empagliflozin, cardiovascular outcomes, and mortality in type 2 diabetes. N. Engl. J. Med. 373, 2117-2128 (2015).

25. Marso, S. P. et al. Liraglutide and cardiovascular outcomes in type 2 diabetes. N. Engl. J. Med. 375, 311-322 (2016).

26. Ratner, R. et al. Cardiovascular safety of exenatide BID: an integrated analysis from controlled clinical trials in participants with type 2 diabetes. Cardiovasc. Diabetol. 10, 22 (2011).

27. Marso, S. P. et al. Cardiovascular safety of liraglutide assessed in a patient-level pooled analysis of phase 2: 3 liraglutide clinical development studies. Diab. Vasc. Dis. Res. 8, 237-240 (2011).

28. Ferdinand, K. C., Botros, F. T., Atisso, C. M. \& Sager, P. T. Cardiovascular safety for once-weekly dulaglutide in type 2 diabetes: a pre-specified meta-analysis of prospectively adjudicated cardiovascular events. Cardiovasc. Diabetol. 15, 38 (2016).

29. Fisher, M. et al. Cardiovascular safety of albiglutide in the Harmony programme: a meta-analysis. Lancet Diabetes Endocrinol. 3, 697-703 (2015).

30. White, W. B. et al. Cardiovascular safety of the dipetidyl peptidase- 4 inhibitor alogliptin in type 2 diabetes mellitus. Diabetes Obes. Metab. 15, 668-673 (2013).

31. Iqbal, N., Parker, A., Frederich, R., Donovan, M. \& Hirshberg, B. Assessment of the cardiovascular safety of saxagliptin in patients with type 2 diabetes mellitus: pooled analysis of 20 clinical trials. Cardiovasc. Diabetol. 13, 33 (2014) 
32. Engel, S. S. et al. Cardiovascular safety of sitagliptin in patients with type 2 diabetes mellitus: a pooled analysis. Cardiovasc. Diabetol. 12, 3 (2013).

33. Johansen, O. E., Neubacher, D., von Eynatten, M., Patel, S. \& Woerle, H. J. Cardiovascular safety with linagliptin in patients with type 2 diabetes mellitus: a pre-specified, prospective, and adjudicated meta-analysis of a phase 3 programme. Cardiovasc. Diabetol. 11, 3 (2012).

34. McInnes, G. et al. Cardiovascular and heart failure safety profile of vildagliptin: a meta-analysis of 17000 patients. Diabetes Obes. Metab. 17, 1085-1092 (2015).

35. Scheen, A. J. Cardiovascular effects of new oral glucose-lowering agents: DPP-4 and SGLT-2 inhibitors. Circ. Res. 122, 1439-1459 (2018)
Publisher's note Springer Nature remains neutral with regard to jurisdictional claims in published maps and institutional affiliations.

(c) Open Access This article is licensed under a Creative Commons

Attribution 4.0 International License, which permits use, sharing, adaptation, distribution and reproduction in any medium or format, as long as you give appropriate credit to the original author(s) and the source, provide a link to the Creative Commons license, and indicate if changes were made. The images or other third party material in this article are included in the article's Creative Commons license, unless indicated otherwise in a credit line to the material. If material is not included in the article's Creative Commons license and your intended use is not permitted by statutory regulation or exceeds the permitted use, you will need to obtain permission directly from the copyright holder. To view a copy of this license, visit http://creativecommons.org/licenses/by/4.0/.

(C) The Author(s) 2022 


\section{Methods}

Trials. This pre-specified meta-analysis of all randomized controlled trials of at least 26-weeks duration from the tirzepatide clinical development program included seven clinical trials: one phase 2 trial, five international phase 3 trials and one regional phase 3 trial in Japan ${ }^{5-7,11,19,36,37}$. Two trials were excluded: one phase 2 trial with 111 participants and 12-weeks duration and one uncontrolled phase 3 safety trial conducted in Japan. Trial durations ranged from 26 to 104 weeks (Supplementary Table 1). Individual data from participants randomized to tirzepatide (1 mg, $5 \mathrm{mg}, 10 \mathrm{mg}$ or $15 \mathrm{mg}$ ) and randomized to placebo or active comparator (insulin degludec, insulin glargine, semaglutide $1 \mathrm{mg}$ or dulaglutide $(1.5 \mathrm{mg}$ or $0.75 \mathrm{mg}))$ were pooled.

Participant population and randomization. The trials included individuals aged 18 years or older with T2D inadequately controlled with diet and exercise with or without metformin, with baseline $\mathrm{HbAlc}$ ranging from $7.0 \%$ to $10.5 \%$ and BMI of $23 \mathrm{~kg} \mathrm{~m}^{-2}$ or higher, depending on the trial. Notably, SURPASS-4 included a high-cardiovascular-risk population and was designed to contribute the majority (that is, approximately 80\%) of the MACE- 4 endpoints for evaluating cardiovascular safety, as compared to the other trials. Participants were randomly assigned 2:1 to tirzepatide (1 mg, $5 \mathrm{mg}, 10 \mathrm{mg}$ or $15 \mathrm{mg}$ ) or comparator (placebo or dulaglutide $1.5 \mathrm{mg}$ ) in the phase 2 trial; $3: 1$ to tirzepatide $(5 \mathrm{mg}, 10 \mathrm{mg}$ or $15 \mathrm{mg}$ ) or comparators (placebo, semaglutide $1 \mathrm{mg}$, insulin degludec or dulaglutide $0.75 \mathrm{mg}$ ) in the phase 3 trials other than SURPASS-4; and 1:1 to tirzepatide $(5 \mathrm{mg}, 10 \mathrm{mg}$ or $15 \mathrm{mg}$ ) or insulin glargine in SURPASS-4 (refs. ${ }^{5-7,11,19,3,6,37}$ ).

Each trial was prospectively registered at ClinicalTrials.gov, received institutional review board approval for each participating center and was conducted in accordance with the Declaration of Helsinki. All participants provided written informed consent for trial participation.

Cardiovascular event adjudication. MACE were prospectively captured and centrally adjudicated using similar event definitions across the trials' program by personnel blinded to randomized assignment by the Duke Clinical Research Institute Clinical Endpoint Committee (CEC) (for the phase 2 trial) and the Cleveland Clinic Coordinating Center for Clinical Research CEC (for the phase 3 trials). The CEC reviewed all deaths (adjudicated cardiovascular and non-cardiovascular) and potential cardiovascular outcome events, including acute coronary syndromes (MI and HUA), coronary revascularization procedures (coronary artery bypass grafting and percutaneous coronary interventions), HHF and cerebrovascular events (stroke and transient ischemic attack).

Outcomes. The primary outcome was time to first occurrence of CEC-confirmed MACE-4 (including cardiovascular death, MI, stroke and HUA). MACE-4 has been used in meta-analyses for several new diabetes medications to exclude excess cardiovascular risk at the time of first regulatory submission as requested by the FDA, EMA and other agencies ${ }^{29,33,38}$. The rationale for inclusion of HUA as a MACE component is driven by the advantage of increased numbers of events ascertained to ensure adequate statistical power for the assessment of cardiovascular safety and was prospectively planned. The secondary outcomes were MACE-3 (including cardiovascular death, MI and stroke), the composite outcome of MACE-3 or $\mathrm{HHF}$ as well as individual MACE components, including cardiovascular death, MI, stroke, HUA, HHF and all-cause death. Secondary outcomes also included the pooled analyses of trials with comparators with anticipated neutral effect on cardiovascular outcomes (insulins ${ }^{18,21}$ ) or placebo combined with best standard of care and the analysis of the trial with a selected high-risk cardiovascular population (SURPASS-4). Additional post hoc analyses included adjudicated outcome of coronary revascularization (urgent and non-urgent and surgical or percutaneous) and the first occurrence of any components of the composite outcome MACE-6 (including MACE-3 and all other adjudicated coronary outcomes (HUA, HHF and revascularization)) as well as subgroup analyses for race, country and SGLT-2i use at baseline.

Statistical analyses. Meta-analysis. The primary aim of this cardiovascular safety meta-analysis, in accordance with FDA and EMA guidance ${ }^{12-15}$, was to demonstrate that tirzepatide was not associated with unacceptably high risk for cardiovascular events versus comparators, defined as an upper bound of the CI of the MACE-4 $\mathrm{HR}<1.8$.

The certainty of evidence obtained from this meta-analysis is high, and there is low basis to downgrade the quality of evidence according to GRADE methodology-based on bias, inconsistency, indirectness, imprecision or publication bias $^{39}$. The intent-to-treat principle, high-quality execution of each of the prospectively randomized trials, the adjudication of endpoints by independent academic groups in a blinded manner and minimal missing data balanced between tirzepatide and comparator arms reduced potential bias (Supplementary Table 5). Plausible bias or indirectness due to the use of cardioprotective comparators, deemed to be minimal, could only bias cardiovascular event safety assessment against tirzepatide compared to control. In addition, the relatively shorter duration of up to 52 weeks of follow-up in clinical trials with low baseline cardiovascular risk compared to up to 104 weeks of follow-up in SURPASS-4 limits assessment for possible inconsistency.
A stratified Cox proportional hazards model was used with treatment (pooled tirzepatide groups and pooled comparator groups) as a fixed effect and stratified trial-level cardiovascular risk (SURPASS-4 and all other trials). This approach preserves the clustering of individual participant data when estimating parameters within strata ${ }^{40}$ and assumes (1) homogeneity of tirzepatide relative treatment effect on MACE regardless of patients' baseline cardiovascular risk level; (2) homogeneity of tirzepatide treatment effect regardless of the comparator in each trial; and (3) proportional hazards of tirzepatide relative to comparator over time.

To account for different randomization ratios for pooled tirzepatide versus comparator between trials, adjusted estimates of means, standard deviations, event rates and percentages were obtained by weighting with inverse probability of randomization for treatment within stratum ${ }^{41}$. Cumulative incidence on time to first event was estimated using the adjusted Kaplan-Meier estimator weighting with inverse probability of randomization for treatment within stratum ${ }^{42}$. A stratified Cox proportional hazards regression model stratified by trial-level cardiovascular risk was also used for each subgroup analysis. The model contained treatment, the subgroup variable and the treatment-by-subgroup interaction term as fixed effects and trial-level cardiovascular risk stratification. All tests of interactions between treatment and subgroup were conducted at a two-sided alpha level of 0.10. Analyses of the following subgroups were pre-specified: sex, age $(<65$ years and $\geq 65$ years) and baseline $\mathrm{HbAlc}(\leq 8.5 \%$ and $>8.5 \%)$. Analyses of other subgroups were planned post hoc, including race (White or Non-White), country (US or non-US) and baseline SGLT-2i use (yes or no). The analyses included all randomized participants receiving at least one treatment dose (modified intent-to-treat (mITT) population), and all analyses were conducted using individual participant data in accordance with the intention-to-treat principle.

With these methods, assuming no difference in cardiovascular risk between tirzepatide and comparators, it was determined that 133 participants with MACE- 4 would provide $90 \%$ power to exclude the upper limit of the $95 \%$ CI of 1.8 at two-sided alpha $=0.05$. It was anticipated that approximately 110 of the 133 MACE- 4 outcomers would be ascertained in the SURPASS- 4 trial, which was designed as an event-driven trial continuing until 133 MACE-4 outcomes were confirmed, pooling results across all the relevant trials. Analyses were conducted using SAS Enterprise Guide version 7.1.

Interim analysis. An interim analysis was performed by an individual data monitoring committee, and the sponsor project members were kept blinded to the treatment assignments until individual trial data were locked. The purpose of the interim cardiovascular meta-analysis was to determine whether the pre-marketing cardiovascular safety requirement for regulatory submission was met. Assuming a $10 \%$ reduced cardiovascular risk with tirzepatide versus comparators, accrual of 100 participants with MACE- 4 would provide $80 \%$ power to discharge an unacceptable increase in cardiovascular risk at approximately a two-sided significance level of 0.01 . An interim meta-analysis was planned when at least 100 participants had experienced one component of MACE-4; when all global phase 3 trials, except SURPASS-4, were completed; and when regulatory-mandated long-term exposure requirements were met. To discharge an unacceptable increase in cardiovascular risk, the upper bound of the CI of the MACE- 4 HR comparing pooled tirzepatide and pooled comparator less than 1.8 should be demonstrated. The alpha level for the interim analysis was to be calculated based on the fraction of 133 planned primary MACE- 4 outcomes available at the interim analysis, with gamma of -6.6 guided by the Hwang-Shih-De Cani method ${ }^{43}$. If an unacceptable increase in cardiovascular risk was discharged at the interim analysis, close-out of the ongoing SURPASS-4 trial was to be initiated, with an anticipated close-out period of 3 months, during which accrual of additional MACE- 4 was possible. Accordingly, the primary cardiovascular risk assessment of tirzepatide comprised the interim analysis, and the subsequent analyses using the final dataset after the completion of SURPASS-4 and SURPASS J-mono were conducted using a nominal significance level of 0.05 ( $95 \% \mathrm{CI})$. If an unacceptable increase in cardiovascular risk was not discharged at the interim analysis, the SURPASS-4 trial was to continue until accrual of 133 participants with MACE- 4 occurred. In this setting, the primary cardiovascular risk assessment of tirzepatide was to be the analysis conducted with all available MACE- 4 at the conclusion of SURPASS- 4 . The alpha level available for the final analysis was to be calculated based on the alpha spent at the interim analysis, the number of participants with MACE- 4 at the interim analysis and the number of participants with MACE- 4 at the final analysis.

\section{Methods accounting for differences in randomization ratios and study-level} cardiovascular risk. Due to the asymmetric randomization ratios and patient populations with different cardiovascular risk across the trials, the crude estimates simply aggregating the trials on each of the pooled tirzepatide group and the pooled comparator group could be misleading. Therefore, only adjusted estimates for each pooled treatment group were presented for summary statistics and event rate and cumulative incidence on time to first outcome. The adjustments were made by weighting with inverse probability of randomization for pooled treatment groups within stratum.

Within each stratum (SURPASS-4 only and all other trials), baseline cardiovascular risk factors were well balanced among the pooled treatment groups (Supplementary Tables 6 and 7), although patients' background of cardiovascular 
risk was different between strata (for example, $86.9 \%$ of participants for SURPASS- 4 only and $10.6 \%$ of participants for all other trials had history of cardiovascular disease). Thus, the crude estimates of percentage were different (for example, $28.9 \%$ of participants in pooled tirzepatide and $47.2 \%$ of participants in pooled comparator had history of cardiovascular disease) (Supplementary Table 8). After adjustment, the estimates were balanced among the pooled treatment groups (for example, $35.1 \%$ of participants in pooled tirzepatide and $34.4 \%$ of participants in pooled comparator had history of cardiovascular disease) (Supplementary Table 8). Adjusted estimates are the adequate values for comparing distribution among the pooled treatment groups.

Reporting Summary. Further information on research design is available in the Nature Research Reporting Summary linked to this article.

\section{Data availability}

Eli Lilly provides access to all individual participant data collected during the trial, after anonymization, with the exception of pharmacokinetic or genetic data. Data are available to request 6 months after the indication studied has been approved in the United States and the European Union and after primary publication acceptance, whichever is later. No expiration date of data requests is currently set once data are made available. Access is provided after a proposal has been approved by an independent review committee identified for this purpose and after receipt of a signed data sharing agreement. Data and documents, including the study protocol, statistical analysis plan, clinical study report and blank or annotated case report forms, will be provided in a secure data sharing environment. For details on submitting a request, see the instructions provided at www.vivli.org. Additional details of each trial assessed in these meta-analyses can be found at http://clinicaltrials.gov as NCT03131687 (phase 2), NCT03954834 (SURPASS-1), NCT03987919 (SURPASS-2), NCT03882970 (SURPASS-3), NCT03730662 (SURPASS-4), NCT04039503 (SURPASS-5) and NCT03861052 (SURPASS J-mono).

\section{References}

36. A Study of Tirzepatide (LY3298176) Compared to Dulaglutide in Participants With Type 2 Diabetes (SURPASS J-mono). https://clinicaltrials.gov/ct2/show/ NCT03861052

37. Dahl, D. et al. Effect of subcutaneous tirzepatide vs placebo added to titrated insulin glargine on glycemic control in patients with type 2 diabetes: the SURPASS-5 randomized clinical trial. JAMA 327, 534-545 (2022).

38. Sonesson, C., Johansson, P. A., Johnsson, E. \& Gause-Nilsson, I. Cardiovascular effects of dapagliflozin in patients with type 2 diabetes and different risk categories: a meta-analysis. Cardiovasc. Diabetol. 15, 37 (2016).

39. Chandler, J. et al. Cochrane Methods. Cochrane Database Syst. Rev. 10 https:// doi.org/10.1002/14651858.CD201601 (2016).

40. Riley, R. D., Lambert, P. C. \& Abo-Zaid, G. Meta-analysis of individual participant data: rationale, conduct, and reporting. BMJ 340, c221 (2010).

41. Horvitz, D. G. \& Thompson, D. J. A generalization of sampling without replacement from a finite universe. J. Am. Stat. Assoc. 74, 663-685 (1952).
42. Xie, J. \& Liu, C. Adjusted Kaplan-Meier estimator and log-rank test with inverse probability of treatment weighting for survival data. Stat. Med. 24, 3085-3110 (2005).

43. Hwang, I. K., Shih, W. J. \& De Cani, J. S. Group sequential designs using a family of type I error probability spending functions. Stat. Med. 9, 1439-1445 (1990).

\section{Acknowledgements}

The authors thank C. A. Karanikas (Eli Lilly and Company) for writing and editorial assistance. This study was supported by Eli Lilly and Company. N.S. is supported by the British Heart Foundation Research Excellence Award (RE/18/6/34217).

\section{Author contributions}

I.P., G.J.W., H.N. and R.J.W. contributed to the statistical analysis plan. R.J.W. was responsible for adjudication across the trials included. G.J.W. and H.N. were responsible for the statistical considerations in the analysis and trial design. I.P. and R.J.W. are the guarantors of this work and, as such, take responsibility for the integrity of the data and the accuracy of the data analysis. All authors participated in critically reviewing and interpreting the data for the manuscript and in revising the submission and approving final submission.

\section{Competing interests}

N.S. has consulted for or received lecture fees from Amgen, AstraZeneca, Boehringer Ingelheim, Eli Lilly and Company, Hanmi Pharmaceuticals, Merck Sharp \& Dohme, Novartis, Novo Nordisk, Pfizer and Sanofi and received grant support from AstraZeneca, Boehringer Ingelheim, Novartis and Roche Diagnostics through his institution, the University of Glasgow. D.K.M. reports personal fees from Boehringer Ingelheim, Janssen Research and Development, Sanofi US, Merck \& Co., Merck Sharp \& Dohme, Eli Lilly and Company, Novo Nordisk, GlaxoSmithKline, AstraZeneca, Lexicon Pharmaceuticals, Eisai, Pfizer, Metavant, Applied Therapeutics, Afimmune, Bayer and Esperion. S.Z. reports participation in advisory boards, expert committees or educational meetings on behalf of Monash University for Eli Lilly and Company, Boehringer Ingelheim, Sanofi, Servier, AstraZeneca, Novo Nordisk and Merck Sharp \& Dohme Australia, outside of the submitted work. N.S., D.K.M. and S.Z. sit on the steering committee for the SURPASS-CVOT. I.P., R.J.W., G.J.W. and H.N. are employees and shareholders of Eli Lilly and Company.

\section{Additional information}

Extended data is available for this paper at https://doi.org/10.1038/s41591-022-01707-4.

Supplementary information The online version contains supplementary material available at https://doi.org/10.1038/s41591-022-01707-4.

Correspondence and requests for materials should be addressed to Naveed Sattar or Sophia Zoungas.

Peer review information Nature Medicine thanks Alice Cheng and the other, anonymous, reviewer(s) for their contribution to the peer review of this work. Jennifer Sargent was the primary editor on this article and managed its editorial process and peer review in collaboration with the rest of the editorial team.

Reprints and permissions information is available at www.nature.com/reprints. 


\section{All Tirzepatide \\ Events/participants $n\left(n / 100\right.$ person-years $\left.{ }^{a}\right)$}

All Comparator Events/participants $n\left(n / 100\right.$ person-years $\left.{ }^{a}\right)$
Hazard Ratio p-value (95\% CI)

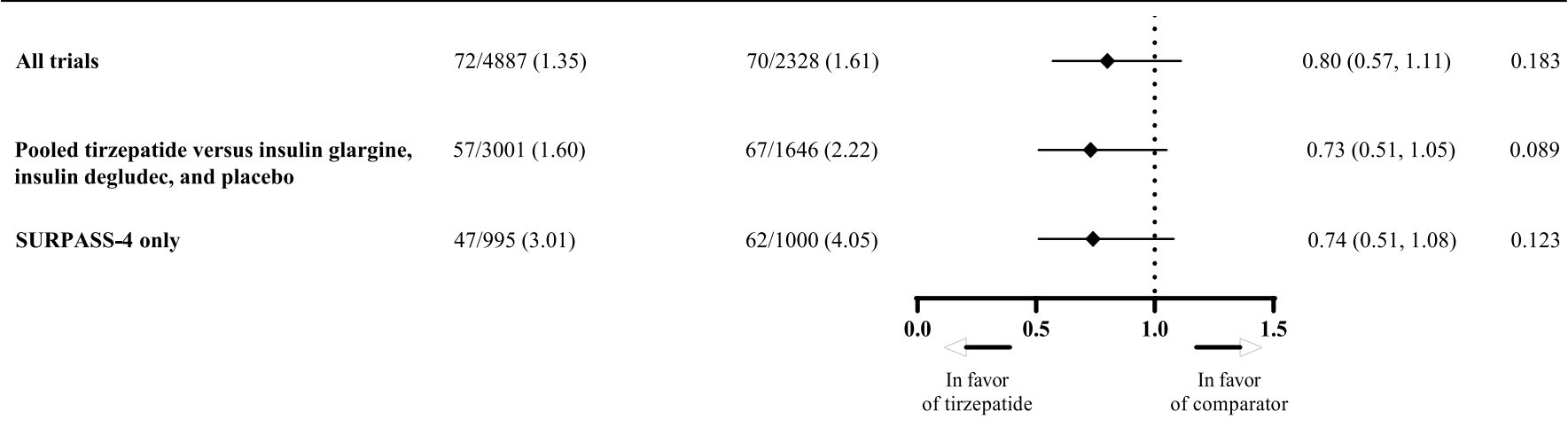

Extended Data Fig. 1 | Time-to-event analyses of composite adjudication-confirmed MACE-4. Data are point estimate of HR (illustrated by the diamond symbol) and range of 2-sided $95 \% \mathrm{Cl}$ of the HR. a Strata size adjusted estimate. Strata are defined as trial-level cardiovascular risk (SURPASS-4 forms one stratum, and all other trials form one stratum). Abbreviations: $\mathrm{Cl}=$ confidence interval; $\mathrm{HR}=$ hazard ratio; iDeg = insulin degludec; iGlar = insulin glargine; $\mathrm{MACE}=$ major adverse cardiovascular events; $\mathrm{n}=$ number of participants in the specified category; $\mathrm{PBO}=\mathrm{placebo} ; \mathrm{TZP}=$ tirzepatide. 


\section{Reporting Summary}

Nature Research wishes to improve the reproducibility of the work that we publish. This form provides structure for consistency and transparency in reporting. For further information on Nature Research policies, see our Editorial Policies and the Editorial Policy Checklist.

\section{Statistics}

For all statistical analyses, confirm that the following items are present in the figure legend, table legend, main text, or Methods section.

$\mathrm{n} / \mathrm{a}$ Confirmed

$\bigotimes$ The exact sample size $(n)$ for each experimental group/condition, given as a discrete number and unit of measurement

\ A statement on whether measurements were taken from distinct samples or whether the same sample was measured repeatedly

The statistical test(s) used AND whether they are one- or two-sided

Only common tests should be described solely by name; describe more complex techniques in the Methods section.

\ A description of all covariates tested

$\square$ \ A description of any assumptions or corrections, such as tests of normality and adjustment for multiple comparisons

A full description of the statistical parameters including central tendency (e.g. means) or other basic estimates (e.g. regression coefficient)

AND variation (e.g. standard deviation) or associated estimates of uncertainty (e.g. confidence intervals)

$\square$ For null hypothesis testing, the test statistic (e.g. $F, t, r$ ) with confidence intervals, effect sizes, degrees of freedom and $P$ value noted

Give $P$ values as exact values whenever suitable.

Х $\square$ For Bayesian analysis, information on the choice of priors and Markov chain Monte Carlo settings

$\square$ \ For hierarchical and complex designs, identification of the appropriate level for tests and full reporting of outcomes

$\triangle(\square$ Estimates of effect sizes (e.g. Cohen's d, Pearson's $r$ ), indicating how they were calculated

\section{Our web collection on statistics for biologists contains articles on many of the points above.}

\section{Software and code}

Policy information about availability of computer code

Data collection No software was used for data collection. Analyses were conducted using SAS Enterprise Guide V7.1.

Data analysis Analyses were conducted using SAS Enterprise Guide V7.1.

For manuscripts utilizing custom algorithms or software that are central to the research but not yet described in published literature, software must be made available to editors and

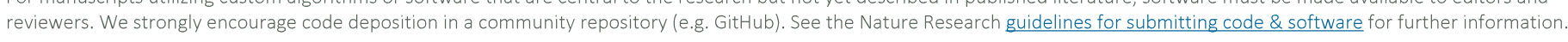

\section{Data}

Policy information about availability of data

All manuscripts must include a data availability statement. This statement should provide the following information, where applicable:

- Accession codes, unique identifiers, or web links for publicly available datasets

- A list of figures that have associated raw data

- A description of any restrictions on data availability

Lilly provides access to all individual participant data collected during the trial, after anonymization, with the exception of pharmacokinetic or genetic data. Data are available to request 6 months after the indication studied has been approved in the US and EU and after primary publication acceptance, whichever is later. No expiration date of data requests is currently set once data are made available. Access is provided after a proposal has been approved by an independent review committee identified for this purpose and after receipt of a signed data sharing agreement. Data and documents, including the study protocol, statistical analysis plan, clinical study report, blank or annotated case report forms, will be provided in a secure data sharing environment. For details on submitting a request, see the instructions provided at www.vivli.org. Additional details of each trial assessed in these meta-analyses can be found at http://clinicaltrials.gov as NCT03131687 (Phase 2), NCT03954834 (SURPASS-1), NCT03987919 (SURPASS-2), NCT03882970 (SURPASS-3), NCT03730662 (SURPASS-4), NCT04039503 (SURPASS-5) and NCT03861052 (SURPASS J-mono). 
Please select the one below that is the best fit for your research. If you are not sure, read the appropriate sections before making your selection.

Х Life sciences

Behavioural \& social sciences

Ecological, evolutionary \& environmental sciences

For a reference copy of the document with all sections, see nature.com/documents/nr-reporting-summary-flat.pdf

\section{Life sciences study design}

All studies must disclose on these points even when the disclosure is negative.

Sample size Determination of sample size was reported in each individual trial included in this meta-analysis. For this meta-analysis and under the assumption of no excess $\mathrm{CV}$ risk with tirzepatide compared with comparators, the sample size was determined for selected combinations of the number of MACE-4 from SURPASS-4 and the number of MACE-4 from other Ph 3 trials combined, leading to statistical power of at least $90 \%$ to demonstrate upper bound of $95 \% \mathrm{Cl}$ for $\mathrm{HR}<1.8$.

Data exclusions Two studies were excluded: one Phase 2 trial with 111 participants and 12 weeks duration and one uncontrolled Phase 3 safety trial conducted in Japan. Trial durations ranged from 26-104 weeks (Supplementary Table 1). Individual data from participants randomized to tirzepatide ( $1 \mathrm{mg}, 5 \mathrm{mg}, 10 \mathrm{mg}$ or $15 \mathrm{mg}$ ) and randomized to placebo or active comparator (insulin degludec, insulin glargine, semaglutide 1 $\mathrm{mg}$, or dulaglutide [ $1.5 \mathrm{mg}$ or $0.75 \mathrm{mg}$ ]) were pooled. Furthermore, this CV meta-analysis included tirzepatide clinical trials that excluded participants with recent unstable CV disease (e.g., the New York Heart Association Class IV heart failure and recent CV events).

Replication Results of these meta-analysis are based on randomized clinical trials conducted in accordance with the Declaration of Helsinki and Good Clinical Practice guidelines with CV outcomes adjudicated by an independent clinical endpoint committee in a blinded fashion.

Randomization Participants were randomly assigned 2:1 to tirzepatide ( $1 \mathrm{mg}, 5 \mathrm{mg}, 10 \mathrm{mg}$ or $15 \mathrm{mg}$ ) or comparator (placebo or dulaglutide $1.5 \mathrm{mg}$ ) in the Phase 2 trial, $3: 1$ to tirzepatide ( $5 \mathrm{mg}, 10 \mathrm{mg}$ or $15 \mathrm{mg}$ ) or comparators (placebo, semaglutide $1 \mathrm{mg}$, insulin degludec or dulaglutide $0.75 \mathrm{mg}$ ) in the Phase 3 trials other than SURPASS-4, and 1:1 to tirzepatide $(5 \mathrm{mg}, 10 \mathrm{mg}$, or $15 \mathrm{mg}$ ) or insulin glargine in SURPASS-4.

Blinding

Blinding was reported in each individual trial included in this meta-analysis and in Supplementary Table 1. In summary, Ph 2 GPGB, SURPASS-1, SURPASS-5 and SURPASS J-mono were double blind and investigators were blinded to group allocation during data collection and analysis. SURPASS-2, -3 and -4 were open-labeled. SURPASS- 2 was an active-controlled study with semaglutide $1 \mathrm{mg}$ and could not be blinded because of differences in devices. SURPASS-3 and -4 were active-controlled studies with insulin and could not be blinded because of differences in dosing schedules and devices. All studies were randomized.

\section{Reporting for specific materials, systems and methods}

We require information from authors about some types of materials, experimental systems and methods used in many studies. Here, indicate whether each material, system or method listed is relevant to your study. If you are not sure if a list item applies to your research, read the appropriate section before selecting a response.

\begin{tabular}{l|l} 
Materials \& experimental syste \\
\hline$n / a$ & Involved in the study \\
\hline & $\square$ Antibodies \\
\hline & $\square$ Eukaryotic cell lines \\
$\searrow$ & $\square$ Palaeontology and archaeology \\
$\square$ & $\square$ Animals and other organisms \\
$\square$ & $\bigotimes$ Clinical data \\
$\square$ & $\square$ Dual use research of concern
\end{tabular}

Methods n/a Involved in the study Х $\square$ Chip-seq

Х $\square$ Flow cytometry

Х $\square$ MRI-based neuroimaging

\section{Human research participants}

Policy information about studies involving human research participants

Population characteristics

The trials included individuals aged $\geq 18$ years with $T 2 \mathrm{D}$ inadequately controlled with diet and exercise \pm metformin, with a baseline $\mathrm{HbA} 1 \mathrm{c}$ ranging from $7.0-10.5 \%$, and $\mathrm{BMI} \geq 23 \mathrm{~kg} / \mathrm{m} 2$, depending on the trial. Importantly, SURPASS-4 included a high $\mathrm{CV}$ risk population and was designed to contribute the majority (i.e., approximately $80 \%$ ) of the MACE-4 endpoints for evaluating $\mathrm{CV}$ safety, as compared with the other studies. The baseline demographic characteristics were balanced across the pooled tirzepatide and pooled comparator group. Overall, participants had a mean a baseline HbA1c $8.3 \%$, a mean diabetes duration of 9.3 years, and a mean BMl of $32.8 \mathrm{~kg} / \mathrm{m} 2$, a mean age of 59 years and $43 \%$ were female. 


\section{Clinical data}

Policy information about clinical studies

All manuscripts should comply with the ICMJE guidelines for publication of clinical research and a completed CONSORT checklist must be included with all submissions.

Clinical trial registration Additional details of trials assessed in these meta-analyses can be found at http://clinicaltrials.gov as NCT03131687 (Phase 2), NCT03954834 (SURPASS-1), NCT03987919 (SURPASS-2), NCT03882970 (SURPASS-3), NCT03730662 (SURPASS-4), NCT04039503 (SURPASS-5) and NCT03861052 (SURPASS J-mono).

Study protocol

Study protocols of all published are trials are provided in the supplemental appendix of the primary manuscript submissions to NEJM (SURPASS-2) and Lancet (SURPASS-1, SURPASS-3, SURPASS-4 and Ph2 GPGB) Protocols of (SURPASS-5 and J-mono) will be available as soon as the corresponding primary manuscripts are published.

Data collection

For the Ph 2 GPGB trial (NCT03131687) data were collected at 47 sites (medical and clinical research centres) in Poland, Puerto Rico, Slovakia, and USA during May 2017 - August 2018. For the SURPASS-1 trial (NCT03954834), data were collected at 52 medical research centers and hospitals in India, Japan, Mexico and the USA during June 2019- October 2020. For the SURPASS-2 trial (NCT03987919), data were collected in 28 sites in the USA, Argentina, Australia, Brazil, Canada, Israel, Mexico, and UK during July 2019-February 2021, For the SURPASS-3 trial (NCT03882970), data were collected in 121 sites in Argentina, Austria, Greece, Hungary, Italy, Poland, Romania, South Korea, Spain, Taiwan, Ukraine, and the United States during April 2019-January 2021. For the SURPASS-4 trial (NCT03730662), data were collected in 187 sites in Argentina, Australia, Brazil, Canada, Greece, Israel, Mexico, Poland, Romania, Russia, Slovakia, Spain, Taiwan, and the USA during November 2018-April 2021. For the SURPASS-5 trial (NCT04039503), data were collected in one center in the USA during August 2019 - January 2021. For the SURPASS J-mono trial (NCT03861052), data were collected in multiple sites in Japan during May 2017 - March 2021. Data were collected using the Electronic Data Capture system.

The primary outcome was time to first occurrence of CEC-confirmed MACE- 4 (including CV death, MI, stroke, and HUA). MACE- 4 has been used in meta-analyses for several new diabetes medications to exclude excess CV risk at the time of first regulatory submission as requested by the FDA, EMA and other agencies. The rationale for inclusion of hospitalization for unstable angina as a MACE component is driven by the advantage of increased numbers of events ascertained to ensure adequate statistical power for the assessment of CV safety and was prospectively planned. The secondary outcomes were MACE-3 (including CV death, MI and stroke), the composite outcome of MACE-3 or HHF, as well as individual MACE components, including CV death, MI, stroke, HUA, HHF, and all-cause death. Secondary outcomes also included the pooled analyses of trials with comparators with anticipated neutral effect on CV outcomes (insulins) or placebo combined with best standard of care) and the analysis of the trial with a selected high risk CV population (SURPASS-4). Additional post-hoc analyses included adjudicated outcome of coronary revascularization (urgent and nonurgent; surgical or percutaneous) and the first occurrence of any components of the composite outcome MACE- 6 (including MACE-3 and all other adjudicated coronary outcomes [HUA, HHF and revascularization]), as well as subgroup analyses for race, country and SGLT-2i use at baseline.

The primary aim of this CV safety meta-analysis, in accordance with the FDA and EMA guidance, was to demonstrate that tirzepatide was not associated with unacceptably high risk for $\mathrm{CV}$ events versus comparators, defined as an upper bound of the confidence interval $(\mathrm{Cl})$ of the 4-component major adverse CV event hazard ratio (MACE-4 HR) $<1.8$.

The certainty of evidence obtained from this meta-analysis is high and there is low basis to downgrade quality of evidence according to GRADE methodology: based on bias, inconsistency, indirectness, imprecision, or publication bias. The intent-to-treat principle, high quality execution of each of the prospectively randomized trials, the adjudication of endpoints by independent academic groups in a blinded manner, and minimal missing data balanced between tirzepatide and comparator arms reduced potential bias (Table S5). Plausible bias or indirectness due to the use of cardioprotective comparators, deemed to be minimal, could only bias CV event safety assessment against tirzepatide compared to control. In addition, the relatively shorter duration of up to 52 weeks follow-up in clinical trials with low baseline CV risk compared to up to 104 weeks of follow-up in SURPASS-4 limits assessment for possible inconsistency. A stratified Cox proportional hazards model was used with treatment (pooled tirzepatide groups, pooled comparator groups) as a fixed effect and stratified trial-level CV risk (SURPASS-4, all other trials). This approach preserves the clustering of individual participant data when estimating parameters within strata. and assumes a) homogeneity of tirzepatide relative treatment effect on MACE regardless of patient's baseline CV risk level; b) homogeneity of tirzepatide treatment effect regardless of the comparator in each trial; and c) proportional hazards of tirzepatide relative to comparator over time.

To account for different randomization ratios for pooled tirzepatide versus comparator between trials, adjusted estimates of means, standard deviations (SD), event rates, and percentages were obtained by weighting with inverse probability of randomization for treatment within stratum. Cumulative incidence on time to first event was estimated using the adjusted Kaplan-Meier estimator weighting with inverse probability of randomization for treatment within stratum. A stratified Cox proportional hazards regression model stratified by trial-level CV risk was also used for each subgroup analysis. The model contained treatment, the subgroup variable, and the treatment-by-subgroup interaction term as fixed effects and trial-level CV risk stratification. All tests of interactions between treatment and subgroup were conducted at a 2-sided alpha level of 0.10. Analyses of the following subgroups were prespecified: sex, age ( $<65$ years, $\geq 65$ years), and baseline $\mathrm{HbA1c}(\leq 8.5 \%,>8.5 \%)$. Analyses of other subgroups were planned post hoc, including race (White, Non-White), country (US, non-US), and baseline SGLT-2i use (yes, no). The analyses included all randomized participants receiving at least one treatment dose (modified intent-to-treat population) and all analyses were conducted using individual participant data in accord with the intention to treat principle. 